\title{
Roles of the plasminogen activator streptokinase and the plasminogen-associated $M$ protein in an experimental model for streptococcal impetigo
}

\author{
Mikael D. Svensson, ${ }^{1}$ Ulf Sjöbring, ${ }^{1}$ Feng Luo ${ }^{2}$ and Debra E. Bessen ${ }^{2}$
}

Author for correspondence: Debra E. Bessen. Tel: +1 203432 7890. Fax: +1 2034326533 . e-mail: debra.bessen@yale.edu

1 Department of Laboratory Medicine, Lund University, Lund, Sweden

2 Department of Ecology and Evolutionary Biology, Yale University, 165 Prospect Street New Haven, CT, USA
Primary infection by group A streptococci (GAS) takes place at either the throat or skin of the human host, often leading to pharyngitis or impetigo, respectively. Many GAS strains differ in their preference for throat and skin tissue sites. Previous epidemiological findings show that many of the strains displaying strong tropism for the skin have a high-affinity binding site for plasminogen, located within M protein (PAM), a prominent surface fibril. Plasminogen bound by PAM interacts with streptokinase, a plasminogen activator secreted by GAS, to yield bacterial-bound plasmin activity. In this study, PAM and streptokinase were tested for their roles in infection using an experimental model that closely mimics human impetigo. Inactivation of genes encoding either PAM or streptokinase led to a partial, but significant, loss of virulence in vivo, as measured by net growth of the bacteria and pathological alterations. The relative loss in virulence in vivo was greater for the streptokinase mutant than for the PAM mutant. However, the PAM mutant, but not the streptokinase mutant, displayed a partial loss in resistance to phagocytosis in vitro. The combined experimental and epidemiological data provide evidence that PAM and streptokinase play a key role in mediating skinspecific infection by GAS. In addition, secreted cysteine proteinase activity due to SpeB leads to degradation of streptokinase in stationary phase broth cultures. Since SpeB is also a determinant of tissue-specific GAS infection at the skin, direct interactions between these two proteolytic pathways may constitute an important pathogenic mechanism. An integrated model for superficial infection at the skin is presented.

Keywords: streptococci, proteolysis, plasmin, inflammation

\section{INTRODUCTION}

Group A streptococci (GAS) are a highly prevalent group of bacterial pathogens that afflict humans. They are well-adapted to superficial epithelial tissue (oropharynx or epidermis), where they can be highly adept at generating progeny that are transmitted to new human hosts. As a consequence of their successful evolutionary strategy, GAS often cause mild disease at

Abbreviations: GAS, group A streptococci; HA, hyaluronic acid; Plg, plasminogen; PMN, polymorphonuclear leukocyte; URT, upper respiratory tract. the throat or skin, in the form of pharyngitis or impetigo, respectively.

Decades of epidemiological findings support the existence of GAS strains that have strong preferences for either the throat or skin, as well as other strains whose tissue site preference is less clear cut (Anthony et al., 1976; Bisno \& Stevens, 2000; Maxted, 1980; Wannamaker, 1970). Thus, there should exist tissuespecific genotypes among GAS strains. The emm pattern is a genetic marker that distinguishes many throat- and skin-tropic strains of GAS (Bessen et al., 1996, 2000; Dicuonzo et al., 2001); this pattern is defined by the chromosomal arrangement of $\mathrm{emm}$ subfamily genes. The emm genes, in turn, encode $M$ surface proteins, 
which display extensive heterogeneity in their structure and function (Cunningham, 2000; Fischetti, 2000). Plasminogen (Plg)-binding group $\underline{A}$ streptococcal $\underline{M}$ protein (PAM) is uniquely associated with $\mathrm{emm}$ pattern D strains (Svensson et al., 1999), which are usually recovered from impetigo lesions and rarely from cases of pharyngitis. Despite their niche separation, there is an ample flow of neutral housekeeping genes between throat and skin strains (Kalia et al., 2002), and high rates of genetic recombination within GAS as a whole (Feil et al., 2001). In instances where neutral housekeeping alleles are randomly distributed with respect to ecologically distinct populations (Kalia et al., 2002), genetic variation that is strongly associated with the different populations may be directly responsible for adaptation to the ecological niche, i.e. emm gene products (or closely linked genes) may have a direct role in tissue tropism. Thus, based on the population genetics findings, a reasonable argument can be made for PAM as a strong candidate for having a tissue-specific role in the development of streptococcal impetigo.

Human Plg can be bound by at least three distinct GAS surface proteins, whereupon it can be converted to its active form (plasmin), through the action of streptokinase, a secreted product of GAS that functions as a Plg activator (Lottenberg et al., 1992; Pancholi \& Fischetti, 1992, 1998). Plasmin acts as a broad-spectrum proteinase. It has been proposed that the acquisition of unregulated surface-associated plasmin by GAS and other bacterial pathogens promotes its spread into deep host tissue (Boyle \& Lottenberg, 1997; Coleman et al., 1997; Rasmussen \& Bjorck, 2002; Sodeinde et al., 1992). However, PAM is distinguished from other GAS Plgbinding proteins in that it has a high affinity for Plg (affinity constant, $8 \times 10^{-8} \mathrm{M}^{-1}$ ) (Berge \& Sjöbring, 1993). The concentration of human Plg in tissue is highest in the circulation and interstitial fluids $\left(1-2 \times 10^{-6} \mathrm{M}\right)$, suggesting that PAM is adapted for functioning in a micro-environment where the $\mathrm{Plg}$ concentration is low. This is consistent with the idea that the interaction of PAM with streptokinase promotes adaptation at a superficial tissue site (i.e. the epidermis).

To test the contribution of specific GAS products to skin infection, an in vivo model was developed that closely mimics impetigo in humans, based on histopathology (Scaramuzzino et al., 2000). Human neonatal foreskins are grafted onto the backs of SCID mice, which fail to reject the xenotransplants. In an effort to mimic natural infection in humans, skin grafts are slightly damaged and GAS are topically applied. The hu-skin-SCID mouse model for impetigo exhibits a high degree of sensitivity, as evidenced by low inoculating doses for virulent strains, and high specificity, whereby emm pattern D strains display greater overall levels of virulence than typical throat strains (emm pattern $\mathrm{A}-\mathrm{C}$ ). Using the huskin-SCID mouse model in conjunction with isogenic mutants of GAS, it can be shown that the secreted cysteine proteinase $\mathrm{SpeB}$ plays a key role in virulence (Svensson et al., 2000). Furthermore, levels of secreted cysteine proteinase activity are highest for $\mathrm{emm}$ pattern
D strains and lowest for emm pattern A-C strains. Thus, experimental findings combined with epidemiological data can provide compelling evidence for the role of a streptococcal product as a determinant of tissue tropism.

In this study, mutants of emm pattern D strain ALAB49 were constructed, whereby the pam gene or the ska gene underwent allelic replacement. The isogenic mutants of ALAB49 were compared to the parent strain for virulence in vivo, using the hu-skin-SCID mouse model for impetigo.

\section{METHODS}

Bacterial culture. The ALAB49 parent strain (ALAB49 wt) was recovered from an impetigo lesion from a patient in Alabama in 1986; it is emm53 and emm pattern D (Enright et al., 2001; Svensson et al., 2000). A streptomycin-resistant variant was selected as described previously (Scaramuzzino et al., 2000; Svensson et al., 2000). GAS were grown in Todd-Hewitt broth with $1 \%$ yeast extract (THY), or on THY/blood agar plates, at $37^{\circ} \mathrm{C}$ for the times indicated. Included in the growth medium were $70 \mu \mathrm{g}$ streptomycin $\mathrm{ml}^{-1}$ plus $200 \mu \mathrm{g}$ kanamycin $\mathrm{ml}^{-1}$ for the mutants (Scaramuzzino et al., 2000; Svensson et al., 2000).

Mutant construction. Directed mutagenesis, via allelic replacement and insertion of the $\Omega \mathrm{Km}-2$ interposon, using the temperature-sensitive shuttle vector pJRS233 harbouring an erythromycin-resistance $\left(\mathrm{Em}^{\mathrm{R}}\right)$ gene, was used to generate $\Delta p a m, \Delta s k a$ and $\Delta s p e B$ mutants of ALAB49 (Caparon, 2000; Kotarsky et al., 2000; Perez-Casal et al., 1993; Ringdahl et al., 1998; Svensson et al., 2000). Plasmid vectors used for GAS gene inactivation were constructed in Escherichia coli. Following electroporation of GAS with purified plasmid DNA, $\mathrm{Em}^{\mathrm{R}}$ transformants were selected by growth on medium containing $15 \mu \mathrm{g}$ erythromycin $\mathrm{ml}^{-1}$. Colony picks of $\mathrm{Em}^{\mathrm{R}}$ transformants were grown to high density in THY broth at $30^{\circ} \mathrm{C}$ and shifted to the non-permissive temperature (in the presence of $200 \mu \mathrm{g} \mathrm{kanamycin} \mathrm{ml}^{-1}$ ), to select for chromosomal integration. Transformants arising from double crossovers were identified by screening for erythromycin sensitivity by replica plating. Construction of the ALAB49 $\Delta s p e B$ mutant has been described in detail already (Svensson et al., 2000). For inactivation of the central $\mathrm{emm}$ gene of ALAB49, encoding PAM (emm53 or pam), two PCR-amplified fragments were prepared, corresponding to the $3^{\prime}$ portion of $m r p 53$ (upstream) through the $5^{\prime}$ portion of $e m m 53$ and to the $3^{\prime}$ portion of emm53 through the $5^{\prime}$ portion of enn53 (downstream). The two PCR fragments were ligated into pJRS233, with $\Omega \mathrm{Km}-2$ introduced between the two PCR fragments, and directed mutagenesis was performed as indicated above. The ALAB49 $\Delta s k a$ mutant was constructed using the replacement vector described for strain AP53 (Ringdahl et al., 1998). This vector was constructed from pJRS233 via ligation of two PCR-amplified fragments corresponding to the $5^{\prime}$ and $3^{\prime}$ portions of the ska gene, which were flanked by the $\Omega \mathrm{Km}-2$ interposon. All mutations were confirmed by PCR-based mapping using the general approach described by Svensson $e t$ al. (2000).

In vivo model for impetigo. The hu-skin-SCID mouse model for streptococcal impetigo was used to measure the virulence of ALAB49 wt and its isogenic mutants (Scaramuzzino et al., 2000; Svensson et al., 2000). Briefly, human neonatal foreskins were grafted onto SCID mice. Healed skin grafts were 
subsequently damaged by a series of gentle, superficial cuts made with a scalpel blade and topically inoculated with $50 \mu \mathrm{l}$ THY broth containing the appropriate concentration of bacteria that had been freshly grown for $24 \mathrm{~h}$ at $37^{\circ} \mathrm{C}$ in THY broth. A wide range of inoculation doses was tested, based on the dilution of the broth cultures. To determine the actual dose administered, duplicate 10-fold dilutions were plated onto agar; duplicate c.f.u. counts were highly concordant with each other and with inoculums used in other experiments. The ratios of c.f.u. to optical density (OD) were consistent for ALAB49 wt and isogenic mutants, suggesting low variation in the mean chain length. At all steps, broth cultures were vigorously vortexed, to break up any aggregates.

Infected human skin grafts were occluded with a bandage. At 7 days post-inoculation, the bandages were removed and the human skin grafts were scored for gross pathology and the animals killed. Following biopsy, each graft was weighed and then split in (approximately) half through the centre with a blade. Half of the graft was used for histopathology and the other half of the graft was used to determine the c.f.u. count. The portion of the graft used for determining the c.f.u. count was reweighed, and bacteria were released from the tissue by vigorous vortexing; final calculations were made by taking a measured portion of the supernatant containing released bacteria, determining the c.f.u. counts following 10-fold serial dilutions (averaged duplicates) on THY/blood agar plates (containing kanamycin for mutant strains) and multiplying the c.f.u. $\mathrm{mg}^{-1}$ by the weight of total tissue biopsied prior to splitting. Spleens were recovered and cultured for the growth of GAS; all mice infected with ALAB49 wt, ALAB49 4 pam and ALAB49 $\Delta s k a$ were negative for septicaemia, with the exception of one spleen which yielded a single colony of GAS. Haematoxylin- and eosin-stained tissue sections were scored, in a blinded fashion, for inflammation and epidermal destruction on a number scale of $0-3$, reflecting the severity of the alterations ( 3 being the most severe). Although gross and microscopic pathology scoring is subjective, it is highly reproducible, even when done in a blinded fashion (Scaramuzzino et al., 2000).

\section{In vitro assays}

Mutants were compared to the ALAB49 wild-type strain for several phenotypic traits that are of relevance to the directed mutagenesis and/or other known virulence properties of GAS.

(i) Bactericidal assay. The Lancefield basic bactericidal test was used to measure antiphagocytic activity, but with bacterial inoculating doses ranging from 150 to 780 c.f.u. $\mathrm{ml}^{-1}$, which more closely reflects the Minneapolis method (Johnson et al., 1996). The ability of GAS to survive and multiply in heparinized human blood following a $3 \mathrm{~h}$ rotation at $37^{\circ} \mathrm{C}$ was assessed by c.f.u. counts. Since this assay measures exponential growth, variance can be large; therefore, to minimize the effects of data skewing, bacteria were tested in four separate experiments, rather than tested as replicates in a single experiment. GAS yielding a 32 -fold increase in c.f.u. values ( 5 population doublings) are regarded as having a completely intact antiphagocytic capacity (Johnson et al., 1996).

(ii) Binding of Plg to bacteria. The binding of radiolabelled Plg, from both human and mouse sources, to whole bacteria was measured, using a standard assay (Ringdahl et al., 1998; Svensson et al., 1999). Heat-killed ALAB49 wt and isogenic mutants were tested over a range of concentrations, generated by serial fivefold dilutions of bacteria. Prior to heat-killing,
GAS were grown to an $\mathrm{OD}_{620}$ value of 0.8 in THY broth, which is equivalent to the late-exponential phase.

(iii) Plasmin acquisition in plasma. Plasmin acquisition by viable ALAB49 wt and the isogenic mutants $\left(10^{10}\right.$ c.f.u. $)$, which were grown overnight and washed with PBS, was assessed by measuring bacterial-bound plasmin following their inoculation into fresh THY broth containing $30 \%$ freshly prepared human plasma, for the times indicated, as described by Ringdahl et al. (1998). Bacterial-bound Plg was eluted with glycine $(\mathrm{pH} 2 \cdot 0)$ and monitored by Western immunoblot using a rabbit antibody specific for human Plg (Dako A/S).

(iv) Streptokinase production. ALAB49 wt and isogenic mutants were grown in THY broth overnight, washed in fresh THY broth and then grown to several OD values reflecting different stages of growth. Bacteria were pelleted by centrifugation and $1 \mathrm{ml}$ of the supernatant was precipitated in $10 \%$ trichloroacetic acid (Svensson et al., 2000). The suspension was incubated on ice for $1 \mathrm{~h}$ and the precipitated proteins were collected by centrifugation. The final pellet was resuspended in SDS sample buffer and analysed by SDS-PAGE and Western blot. The immunoblots were incubated with polyclonal rabbit antibodies raised against purified streptokinase derived from the group $\mathrm{C}$ streptococcal strain H46A, generously provided by Dr Horst Malke (Friedrich Schiller University, Jena, Germany). Streptase (highly purified streptokinase from group C streptococci) was purchased from Hoechst Marion Roussel $\mathrm{AB}$.

(v) Capsule content. The ALAB49 wild-type strain and isogenic mutants were grown to exponential phase, and their hyaluronic acid (HA) capsule contents were measured using Stains-All (Sigma) as described by Ashbaugh \& Wessels (2001). Overnight THY broth cultures of bacteria were diluted $1: 100$ in fresh THY broth and grown to an $\mathrm{OD}_{600}$ value of between $0 \cdot 2$ and 0.3 (mid-exponential phase), prior to HA extraction. C.f.u. counts were determined in duplicate and data were expressed as c.f.u. (fg HA $)^{-1}$, based on a standard curve generated with known quantities of bovine HA.

Statistical analyses. Statistical tests were performed using STATISTICA 5.5 (StatSoft). Tests included the $t$-test for independent samples (unpaired, two-tailed), the non-parametric Mann-Whitney U-test, Fisher's exact test for independence (two-tailed) and Pearson's correlation coefficient.

\section{RESULTS}

\section{In vitro effects of ska and pam inactivation}

Allelic replacement of the $s k a$ and pam genes of strain ALAB49 with the $\Omega \mathrm{Km}-2$ cassette resulted in several phenotypic changes, as measured in vitro. The heatkilled mutant and wild-type organisms were tested over a wide range of concentrations for binding of radiolabelled human Plg (Fig. 1). At a high, but subsaturating, concentration of bacteria, ALAB49 wt and the ALAB49 $\Delta s k a$ mutant bound nearly equivalent amounts of human $\mathrm{Plg}(\sim 80 \%)$; titration of bacteria showed similar binding patterns for the wild-type strain and the $\Delta s k a$ mutant over a range of concentrations. In contrast, the ALAB49 $\Delta$ pam mutant displayed a near-complete loss in binding, even at the highest bacterial concentration tested ( $\sim 5 \%$ bound) (Fig. 1a). The low-level binding of Plg by the $\Delta$ pam mutant is probably due to low-affinity Plg-binding proteins, such as $\alpha$-enolase or 

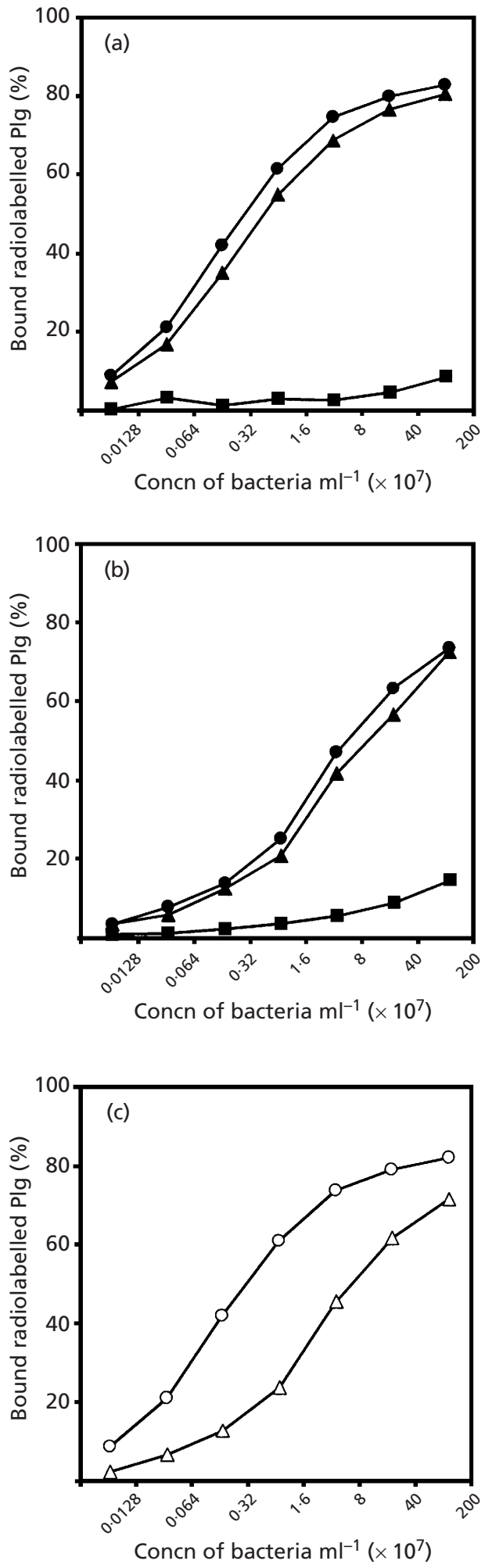

Fig. 1. Binding of human- and mouse-derived Plg by whole bacteria. (a, b) Heat-killed bacteria were tested over a wide range of concentrations for adsorption of ${ }^{125}$ I-labelled Plg derived from human (a) and mouse (b) plasma. Shown are

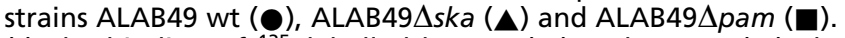
(c) The binding of ${ }^{125}$ I-labelled human $(O)$ and mouse $(\triangle)$ Plg to ALAB49 wt is compared directly.

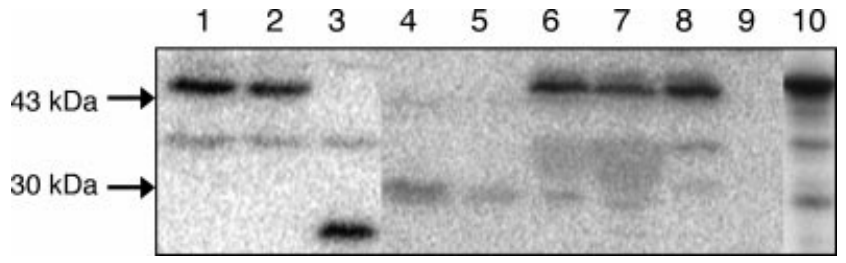

Fig. 2. Immunoblot of streptokinase in bacterial culture supernatants. Late-exponential (lanes 1-3) and stationary (lanes 4-9) phase cultures of ALAB49 wt (lanes 1, 4, 6), ALAB49 $\Delta$ pam (lanes 2, 5, 7), ALAB49 $\Delta$ ska (lanes 3 and 9) and ALAB49 $\Delta$ spe $B$ (lane 8) are shown. The cysteine proteinase inhibitor E64 was added to some bacterial cultures during their growth (lanes 6 and 7). Late-exponential cultures were grown to an $\mathrm{OD}_{620}$ value of 0.8 in THY broth, whereas stationary phase cultures were grown overnight (14-16 h). Purified recombinant streptokinase derived from group $\mathrm{C}$ streptococci (Streptase) was included in lane 10.

glyceraldehyde-3-phosphate dehydrogenase (GAPDH) (Lottenberg et al., 1992; Pancholi \& Fischetti, 1992, 1998). When live bacteria were used in place of heatkilled bacteria, ALAB49 wt bound a high proportion of total Plg, whereas the $\Delta$ pam mutant bound Plg at a very low level (data not shown).

Under similar experimental conditions, with subsaturating concentrations of bacteria, ALAB49 wt and the $\Delta s k a$ mutant bound $\sim 62 \%$ of mouse Plg, whereas ALAB49 4 pam bound only $8 \%$ (Fig. 1b). A direct comparison of binding of human versus mouse Plg by ALAB49 wt is shown in Fig. 1(c). Over a range of bacterial concentrations, mouse Plg is bound about 10to 20-fold less effectively than human Plg. The fact ALAB49 wt can bind mouse Plg reasonably well may be important for the hu-skin-SCID mouse model, whereby mouse Plg is present in extravasated plasma that is dragged by polymorphonuclear leukocytes (PMNs) as they migrate from the circulation to the site of infection. Nonetheless, the presence of human Plg within the human skin graft, or its synthesis in situ upon stimulation by damage or bacteria, cannot be ruled out. PAM binds human Plg with an affinity constant of $8 \times 10^{-8} \mathrm{M}^{-1}$ (Berge \& Sjöbring, 1993), and the concentration of Plg in the circulation and interstitial fluids is $1-2 \times 10^{-6} \mathrm{M}$.

The level of streptokinase production by the mutant and wild-type organisms was monitored by Western immunoblot, using a polyclonal antiserum directed against streptokinase (Fig. 2). Following growth to lateexponential phase, culture broth supernatants derived from both ALAB49 wt and the ALAB49 $\Delta$ pam mutant revealed a strongly immunoreactive band at $44 \mathrm{kDa}$ (Fig. 2, lanes 1 and 2), which corresponded to the expected size for streptokinase. In contrast, the ALAB49 $4 s k a$ mutant lacked a $44 \mathrm{kDa}$ band, but instead displayed a strongly immunoreactive band of $27 \mathrm{kDa}$ (Fig. 2, lane 3), consistent with the expected size for the truncated gene product. 


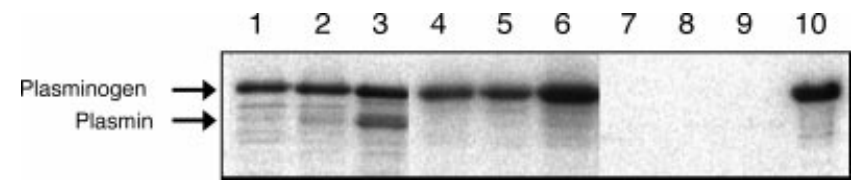

Fig. 3. Immunoblot showing acquisition of Plg and plasmin by wild-type and mutant bacteria. Bacterial-bound proteins eluted from ALAB49 wt (lanes 1-3), ALAB49 $\Delta$ ska (lanes 4-6) and ALAB49 $\Delta$ pam (lanes 7-9) following addition of human plasma and subsequent incubation at $37^{\circ} \mathrm{C}$ for 0 (lanes 1, 4, 7), 30 (lanes 2, 5, 8) and 60 (lanes 3, 6, 9) min are shown. Two micrograms of purified human Plg was included in lane 10.

Acquisition of bacterial-bound plasmin was also measured for ALAB49 wt and the mutant organisms (Fig. 3). Both ALAB49 wt and the ALAB49 $\Delta$ ska mutant bound human Plg. The wild-type strain, but not the $\Delta s k a$ mutant, displayed conversion of Plg to plasmin following $60 \mathrm{~min}$ incubation in $30 \%$ human plasma in THY broth, resulting in an immunoreactive band of a lower molecular mass (Fig. 3, lane 3), following elution from the bacteria and analysis by Western immunoblot using an antibody directed against plasmin(ogen). The ALAB49 $\Delta$ pam mutant failed to bind detectable levels of Plg (Fig. 3, lanes 7-9). Data on Plg binding by live ALAB49 wt and mutant strains (Fig. 3) are consistent with findings on heat-killed bacteria (Fig. 1). The plasmin acquisition assay using mouse plasma as a source was not conducted due to the extreme difficulty in obtaining mouse plasma devoid of even a small degree of activation of the coagulation-fibrinolytic pathways, without the addition of protease inhibitors.

It has long been recognized that the emm gene products of GAS can contribute to the ability of these organisms to resist opsonophagocytosis in the absence of immune sera (Cunningham, 2000; Scott et al., 1986). Following rotation in whole human blood, the ALAB49 $\Delta$ ska mutant is equally resistant to phagocytosis when compared to ALAB49 wt (Table $1 ; t=$ non-significant, $t$-test, unpaired two-tailed). Both the ALAB49 $\Delta s k a$ mutant and ALAB49 wt show mean net increases in their c.f.u. values that are greater than 200 -fold ( $>7$ population doublings), exceeding the 32 -fold threshold (5 popu- lation doublings) which defines survival due to an antiphagocytic effect (Johnson et al., 1996). In contrast, the ALAB49 $\Delta$ pam mutant displays a significant loss in survival relative to the wild-type $(t=0 \cdot 038)$. However, the $\Delta$ pam mutant shows a mean net increase in its c.f.u. counts of 21 -fold ( $2 \cdot 9$ population doublings), indicative of partial and incomplete growth attenuation in human blood; overall, the ALAB49 $\Delta$ pam mutant exceeded the five-population doubling threshold in only one of the four experiments. The finding of partial attenuation for the ALAB49 4 pam mutant is not surprising since, for strains having multiple emm loci, each $\mathrm{emm}$ gene product contributes partially to the antiphagocytic effect (Husmann et al., 1995; Podbielski et al., 1996). Although the range of inoculum doses is slightly lower for ALAB49 4 pam, differences in inoculums for ALAB49 and each mutant are not statistically significant.

In addition to $M$ protein, the HA capsule of GAS can promote resistance to phagocytosis by $\mathrm{PMNs}$, as well as affect GAS adherence to keratinocytes (Darmstadt et al., 2000; Schrager et al., 1998). To better ensure that unintended mutations were not selected for following directed mutagenesis, ALAB49 wt was compared to the $\Delta s k a$ and $\Delta$ pam mutants for HA content (Ashbaugh \& Wessels, 2001). Following growth to mid-exponential phase, differences in $\mathrm{HA}$ content [c.f.u. $(\mathrm{fg} \mathrm{HA})^{-1}$ ] between wild-type and mutant organisms were very slight $[20 \cdot 5,19 \cdot 5 \text { and } 19 \cdot 3 \text { c.f.u. (fg HA })^{-1}$ for wild-type, $\Delta s k a$ and $\Delta p a m$, respectively, measured in duplicate and averaged]. The data suggest that the mutagenesis procedure did not lead to the selection of mutants with alterations in their capsule contents. Additional calculations show that the number of c.f.u. per millilitre of THY broth culture at $\mathrm{OD}_{600}$ was $3 \cdot 5,3 \cdot 8$ and $3 \cdot 1 \times 10^{9}$, respectively, for the wild-type, $\Delta s k a$ and $\Delta$ pam strains. Since optical density measures turbidity, which is independent of variations in chain length, there is strong evidence that the mutations do not affect streptococcal chain length.

Growth curves in THY broth, measured at $\mathrm{OD}_{620}$, were nearly identical for ALAB49 wt and the $\Delta s k a$ and $\Delta p a m$ mutants (data not shown). In addition, the wild-type and the mutant constructs produced equivalent amounts

Table 1. Bacterial growth in whole human blood and the ability to resist phagocytosis

\begin{tabular}{|c|c|c|c|c|c|c|c|c|c|}
\hline \multirow[t]{2}{*}{ Strain } & \multirow{2}{*}{$\begin{array}{l}\text { Range of } \\
\text { inoculums } \\
\text { (c.f.u. } \mathrm{ml}^{-1} \text { ) }\end{array}$} & \multirow[t]{2}{*}{$\begin{array}{l}\text { Mean inoculum } \\
\quad \text { (c.f.u. } \mathrm{ml}^{-1} \text { ) }\end{array}$} & \multirow{2}{*}{$\begin{array}{l}t \text {-Test (versus } \\
\text { wild-type), for } \\
\text { inoculums }\end{array}$} & \multicolumn{5}{|c|}{$\begin{array}{l}\text { Growth following } 3 \mathrm{~h} \text { rotation in } \\
\text { blood (log two-fold increase)* }\end{array}$} & \multirow{2}{*}{$\begin{array}{c}t \text {-Test (versus } \\
\text { wild-type), for } \\
\text { growth }\end{array}$} \\
\hline & & & & Expt 1 & Expt 2 & Expt 3 & Expt 4 & Mean & \\
\hline ALAB49 wt & $460-780$ & 600 & NA & $8 \cdot 325$ & $8 \cdot 616$ & $6 \cdot 370$ & $6 \cdot 677$ & $7 \cdot 497$ & NA \\
\hline ska mutant & $350-740$ & 505 & NS & $7 \cdot 800$ & $7 \cdot 827$ & $7 \cdot 078$ & $7 \cdot 657$ & $7 \cdot 591$ & NS \\
\hline pam mutant & $150-570$ & 375 & NS & $4 \cdot 700$ & $5 \cdot 810$ & $1 \cdot 202$ & $0 \cdot 000$ & $2 \cdot 928$ & $0 \cdot 038$ \\
\hline
\end{tabular}

NA, Not applicable; Ns, non-significant.

*Experiments 1-4 utilized a total of three different blood donors. 
Table 2. Gain or loss in bacterial counts following inoculation of hu-skin-SCID mice

\begin{tabular}{|c|c|c|c|c|c|}
\hline \multicolumn{2}{|c|}{ ALAB49 wt } & \multicolumn{2}{|c|}{ pam mutant } & \multicolumn{2}{|c|}{ ska mutant } \\
\hline $0 \cdot 018$ & $10 \cdot 92$ & 0.024 & $10 \cdot 63$ & 0.076 & $-13 \cdot 29$ \\
\hline $0 \cdot 023$ & 11.99 & 0.035 & $7 \cdot 23$ & $0 \cdot 280$ & -5.62 \\
\hline $0 \cdot 089$ & $8 \cdot 51$ & $0 \cdot 165$ & $-6 \cdot 41$ & 0.343 & $-16 \cdot 61$ \\
\hline \multirow[t]{2}{*}{$2 \cdot 700$} & $2 \cdot 10$ & $3 \cdot 050$ & $-8 \cdot 04$ & $5 \cdot 363$ & $0 \cdot 10$ \\
\hline & & $9 \cdot 050$ & $2 \cdot 3$ & & \\
\hline \multirow{2}{*}{\multicolumn{2}{|c|}{$t$-Test (two-tailed) versus ALAB49 wt }} & $0 \cdot 4514$ & $0.0393^{*}$ & 0.5324 & $0 \cdot 0067 \dagger$ \\
\hline & & NS & & NS & \\
\hline
\end{tabular}

Ns, Non-significant.

"Significant.

† Highly significant. 
of the mature $28 \mathrm{kDa}$ form of $\mathrm{SpeB}$ in late-stationary phase culture supernatants, and displayed complete conversion of SpeB from the $40 \mathrm{kDa}$ zymogen form (data not shown). Despite the strongly polar nature of $\Omega \mathrm{Km}-2$, evidence for decreased expression of downstream genes in our mutants is lacking. The ska transcript is monocistronic (Malke, 2000; Malke et al., 2000) and, based on the SF370 (M1) genome (Ferretti et al., 2001), the nearest downstream gene is $4.5 \mathrm{~kb}$ away (SPy1984, encoding a putative ribonucleotide reductase, $\mathrm{NrdI}$ ) ; intervening ORFs are transcribed on the opposite strand, and rel shares a bidirectional transcriptional terminator with ska (Malke, 2000; Malke et al., 2000). Using gene-specific primers, the relative chromosomal arrangements of ska (SPy1979), rel (SPy1981) and nrdI (SPy1984) were found to be similar for ALAB49 and SF370 (data not shown), unlike the gene order observed for some strains of Streptococcus dysgalactiae subsp. equisimilis (Geyer \& Schmidt, 2000). emm genes are also transcribed monocistronically in several GAS strains that have multiple $\mathrm{emm}$ genes (Bessen \& Fischetti, 1992; Podbielski et al., 1995; Yung \& Hollingshead, 1996).

In summary, phenotypic analysis of the $\Delta s k a$ and $\Delta p a m$ mutants constructed in ALAB49 revealed that each mutant exhibits the changes that were expected, but neither mutant has measurable alterations in several unrelated properties that may be key for virulence. As expected, the ALAB49 sska mutant is unchanged in its $\mathrm{Plg}$ binding, resistance to non-immune phagocytosis, HA capsule and SpeB contents and growth in THY broth; however, it fails to secrete streptokinase and fails to convert surface-bound Plg to plasmin. The ALAB49 $\Delta$ pam mutant is unchanged in its streptokinase and SpeB production, HA capsule content and growth curve but, as expected, shows loss in binding Plg and loss in the antiphagocytic effect, properties that are both attributed to the M53 protein (i.e. PAM).

\section{In vivo effects of ska and pam inactivation}

The ALAB49 $\Delta s k a$ and $\Delta$ pam mutants were tested for virulence in the hu-skin-SCID mouse model for impetigo, over a wide range of inoculating doses. In this model, virulence can be defined as a function of the increase in c.f.u. counts following topical inoculation of GAS onto slightly damaged (scratched) human skin grafts. The 7 day time point chosen for skin biopsy is based on empirical evidence showing a large net growth of virulent strains and histopathological alterations that closely mimic a late stage of the human disease (Scaramuzzino et al., 2000; Svensson et al., 2000). The hu-skin-SCID mouse model is technically challenging, and there is a limit to the number of graft biopsies that can be processed at any one time. Furthermore, since the inoculum doses, expressed as c.f.u. values, are only known in retrospect, it is not possible to test two different constructs at precisely the same dose using the highly accurate c.f.u. measure. Given these constraints, we chose an experimental design whereby each graft receives a different inoculating dose, spanning a wide range of doses, rather than multiple grafts receiving only one or two identical doses. This approach further ensures against overlooking a critical threshold dose, which may differ for the wild-type and each mutant. In natural infections, inoculating doses are likely to fall within a wide range and be dependent on the degree of direct contact and various risk factors, such as skin integrity.

For skin graft biopsies taken at 7 days post-inoculation (Table 2), each mutant showed a partial attenuation in virulence, relative to ALAB49 wt. There were no significant differences for the ranges of inoculating doses tested for ALAB49 wt versus each mutant (Table 2). The difference between ALAB49 wt and each of the mutants in terms of the two-log change in c.f.u. counts (i.e. doubling or halving of the bacterial population) was statistically significant $(t<0 \cdot 05$; unpaired, two-tailed). Although the $\Delta s k a$ mutant showed greater attenuation than the $\Delta$ pam mutant $(t=0.0067$ and 0.0393 , respectively), the $\Delta s k a$ versus $\Delta p a m$ mutants failed to show significant differences relative to one another. Using a non-parametric statistical test (Mann-Whitney $U$-test), loss of virulence for the $\Delta s k a$ mutant was highly significant $(P<0 \cdot 001)$, whereas the $\Delta$ pam mutant showed no significant difference relative to ALAB49 wt. Thus, it appears that the $\Delta s k a$ mutant is highly attenuated for virulence, whereas the $\Delta$ pam mutant is attenuated to a lesser degree. However, the $\Delta$ pam mutant exhibited net reproductive growth for only $44 \%$ of the inoculated skin grafts, in contrast to $100 \%$ of the grafts infected with ALAB49 wt $(P=0 \cdot 029$, Fisher's exact test, two-tailed). If only half of the infections caused by PAM-deficient strains are productive, there may be a significant impact on transmission dynamics (Anderson, 1998). Although statistical analysis strongly supports partial attenuation in virulence resulting from the ska and pam mutations, the loss in virulence does not follow a strict dose-response for either mutant (Table 2); this may be partly due to microscopic graftto-graft variation in the depth of the skin surface scratches, a procedure that is difficult to control with precision and which can on occasion extend beyond the epidermis into the dermal layer (Scaramuzzino et al., 2000).

Gross pathology was scored for grafts infected with ALAB49 wt and each of the mutants, using a relative scale measuring severity of infection - a high score of 3 indicates a purulent exudate (pus) (Scaramuzzino et al., 2000; Svensson et al., 2000). The gross pathology score for the $\Delta s k a$ and $\Delta p a m$ mutants was significantly different when compared to ALAB49 wt (Table 3; all $P$ values $<0 \cdot 02)$. Virulence, as defined by a net gain or loss in c.f.u. values, is generally consistent with the severity of infection, as defined by grossly visible alterations in the skin graft.

Previous histopathological analysis shows that topical inoculation of skin grafts with ALAB49 wt and other virulent GAS strains typically leads to loss of the stratum 
Table 3. Gross and microscopic (blinded review) pathological changes in human skin grafts at 7 days post-inoculation with GAS

Scores for tissue alterations: 0 , no change; 1 , mild changes; 2, moderate changes; 3 , severe changes (Scaramuzzino et al., 2000).

\begin{tabular}{|c|c|c|c|}
\hline \multirow[t]{2}{*}{ Gross and pathological parameters } & \multicolumn{3}{|c|}{ Strain } \\
\hline & ALAB49 wt & pam mutant & ska mutant \\
\hline \multicolumn{4}{|l|}{ Mean score for } \\
\hline Gross pathology & $2 \cdot 750$ & $1 \cdot 389$ & 0.500 \\
\hline Inflammation & $1 \cdot 875$ & $1 \cdot 111$ & $0 \cdot 375$ \\
\hline Epidermal destruction & $1 \cdot 625$ & $0 \cdot 667$ & $0 \cdot 063$ \\
\hline \multicolumn{4}{|c|}{$t$-Value (unpaired, two-tailed $t$-test) versus wild-type, for } \\
\hline Gross pathology & $\mathrm{NA}$ & $0 \cdot 0148 *$ & $0.0005+$ \\
\hline Inflammation & NA & NS & $0.0265^{*}$ \\
\hline Epidermal destruction & $\mathrm{NA}$ & NS & $0.0176^{*}$ \\
\hline \multicolumn{4}{|c|}{$P$-value (Mann-Whitney $U$-test) versus wild-type, for } \\
\hline Gross pathology & $\mathrm{NA}$ & $0 \cdot 0105^{*}$ & $0.0006+$ \\
\hline Inflammation & $\mathrm{NA}$ & NS & $0 \cdot 0250^{*}$ \\
\hline Epidermal destruction & NA & NS & $0 \cdot 0250^{*}$ \\
\hline
\end{tabular}

NA, Not applicable; Ns, non-significant.

*Significant.

† Highly significant.

corneum (keratinized layer) over large sections of skin, infiltration of numerous PMNs throughout the dermis, epidermis and external surface of the skin, and epidermal destruction over large areas of skin. These events have been described in extensive detail, along with accompanying histopathology photographs (Scaramuzzino et al., 2000; Svensson et al., 2000). Similar patterns of histopathological alterations were observed in this study. Six of the seven grafts infected with ALAB49 wt and having a grossly visible purulent exudate on the skin surface at 7 days post-inoculation also scored positive for inflammatory cells present within the tissue, on the external surface and/or in distal foci (Scaramuzzino et al., 2000), according to a microscopic histopathological analysis performed in a blinded fashion (Table 3). The degree of inflammation was lower for grafts inoculated with either the $\Delta$ pam or the $\Delta s k a$ mutant, with statistically significant differences when $\Delta s k a$ is compared to ALAB49 wt (Table 3). A similar trend was noted for destruction of the epidermal layer (Scaramuzzino et al., 2000), whereby the $\Delta$ pam and $\Delta s k a$ mutants showed less epidermal destruction than the wild-type, and the difference between the $\Delta s k a$ mutant and the wild-type was statistically significant. The gross and histopathological findings support the conclusion that the $\Delta s k a$ and $\Delta p a m$ mutants are partially attenuated in virulence, relative to ALAB49 wt, with the $\Delta s k a$ mutant showing a greater loss in virulence than the $\Delta$ pam mutant.

When considering all grafts inoculated with GAS in this study $(n=25)$, the correlation between gross pathology score and the sum of the scores for inflammation and epidermal destruction is statistically significant $(r=0.801$; Pearson's correlation coefficient $)$. Grafts showing a net increase in c.f.u. counts at 7 days postinoculation (Table 2), as compared to grafts showing a net decrease in c.f.u. counts, reveal differences in gross pathology scores that are highly significant $(t=0 \cdot 00027)$. Grafts showing a net increase or decrease in c.f.u. counts at 7 days post-inoculation also showed a strong correspondence with histopathological scores for epidermal destruction and inflammation $(t=0.0036$ and 0.0008 , respectively). The latter finding is consistent with the idea that bacterial reproduction in tissue depends on a source of nutrients provided by extravasated inflammatory cells and that the inflammatory response and/or replicating bacteria contribute to destruction of the epidermal layer.

The hu-skin-SCID mouse model measures the c.f.u. values for bacteria released from the skin graft surface. However, in severely infected tissue, dermal ulcers packed with bacteria can arise; this is characteristic of ecthyma observed in human disease (Scaramuzzino et al., 2000). Therefore, the number of c.f.u. recovered from heavily infected tissue in the hu-skin-SCID mouse model may be an underestimate of the total bacteria associated with the skin graft. Since the wild-type strain yields the most extensive tissue damage (Table 3), it is possible that the net increase in bacterial growth within the total skin graft is higher than reported for vortexreleased bacteria (Table 2). Nonetheless, in natural infections in humans, bacteria present on the superficial skin surface are the most likely to undergo transmission to a new host. 


\section{Interactions of streptokinase and PAM with SpeB}

In a previous study, it was shown that the secreted cysteine proteinase SpeB is critical for GAS infection in the hu-skin-SCID mouse model for impetigo (Svensson et al., 2000). Other investigators have reported that the M1 protein facilitates the conversion of the zymogen form of SpeB to the mature form (Collin \& Olsen, 2000). In contrast, the M53 protein (i.e. PAM) of the ALAB49 strain has no noticeable effect on SpeB conversion. Using culture broth conditions used to prepare inoculums for hu-skin-SCID mice ( $24 \mathrm{~h}$ growth in THY broth), there was complete conversion of SpeB to the mature form, and near-equivalent levels of SpeB for the $\Delta$ pam mutant and ALAB49 wt (data not shown). Also, there were no obvious differences in SpeB production by the ALAB49 $\Delta$ ska mutant.

Cysteine proteinase activity due to SpeB can result in the degradation of M proteins (Berge \& Björck, 1995; Raeder et al., 1998). Studies show that SpeB activity is maximally expressed during the stationary phase of growth (Collin \& Olsen, 2000; Lyon et al., 1998), whereas Mga-regulated emm transcription peaks during the exponential phase (McIver \& Scott, 1997). Control experiments show that the ALAB49 $\Delta s p e B$ mutant (Svensson et al., 2000) binds human Plg at levels equivalent to those measured for ALAB49 wt and ALAB49 $\Delta s k a$, following growth in THY broth to lateexponential phase (Fig. 1a; data not shown). The ALAB49 $\Delta s p e B$ mutant shows no change in antiphagocytic activity in the bactericidal assay (Svensson et al., 2000), consistent with the presence of functionally intact PAM in ALAB49 wt. Thus, under the set of in vitro conditions tested, there is no evidence for modulation of PAM by SpeB in ALAB49 wt nor is there any indication that the loss of PAM influences SpeB activity. Nonetheless, we cannot rule out the possibility that SpeB has an effect on PAM under other experimental conditions (such as stationary phase) or that SpeB modulates the low-affinity Plg-binding surface proteins of ALAB49.

The production of streptokinase by ALAB49 wt and the ALAB49 $\Delta$ pam mutant was monitored by Western immunoblot analysis of culture broth supernatants (Fig. 2 ). Streptokinase was evident in late-exponential phase cultures as a strong $44 \mathrm{kDa}$ band (Fig. 2, lanes 1 and 2), but was significantly degraded in overnight cultures (Fig. 2, lanes 4 and 5). However, addition of the cysteine proteinase inhibitor E64 led to an accumulation of streptokinase in stationary phase cultures (Fig. 2, lanes 6 and 7). That SpeB degrades streptokinase is further supported by findings with overnight culture supernatants of the ALAB49 $\Delta$ speB mutant grown in the absence of E64, which yielded ample quantities of nondegraded streptokinase (Fig. 2, lane 8). The finding of degradation of Ska by SpeB is consistent with an earlier report (Johnston \& Zabriskie, 1986).

Mid-exponential phase cultures of ALAB49 wt and ALAB49 $\Delta$ pam, grown to $\mathrm{OD}_{620}$ values of $0 \cdot 4$, each reveal an immunoreactive band that is somewhat less intense than that observed for late-exponential cultures (data not shown). Streptokinase production was unaffected by the presence of the E64 inhibitor during growth. This finding is consistent with the extracellular production of streptokinase beginning at or before the mid-exponential phase, and streptokinase accumulating throughout the later stages of exponential growth.

In summary, there is no evidence that the inactivation of pam or ska results in alterations in the production of the mature form of SpeB. However, the accumulation of SpeB in stationary phase broth cultures leads to the degradation of extracellular streptokinase in the ALAB49 wild-type strain.

\section{DISCUSSION}

The primary impetus for this study was the observation that GAS strains containing PAM are exclusively emm pattern $\mathrm{D}$; furthermore, pattern $\mathrm{D}$ strains are disproportionately associated with impetigo lesions [versus the upper respiratory tract (URT)] in human hosts (Bessen et al., 1996, 2000; Svensson et al., 1999). In vitro studies demonstrate an interaction between streptokinase and PAM in the generation of bacterial-bound plasmin activity (Ringdahl et al., 1998); therefore, bacterial-bound plasmin may be a determinant for GAS infection at the skin. The findings of this study provide direct experimental evidence that both streptokinase and PAM contribute to virulence during GAS impetigo. When considered together, the experimental and population (epidemiological) findings provide strong evidence that streptokinase and PAM contribute to the establishment of tissue tropism for the skin. Thus, in addition to secreted cysteine proteinase activity due to SpeB (Svensson et al., 2000), the streptokinase-PAM interaction appears to represent a second extracellular proteolytic pathway that is critical for adaptation of GAS at the epidermis. Both SpeB and plasmin function as broad-spectrum proteases.

In the hu-skin-SCID mouse model for impetigo, ALAB49 wt and its mutants exhibit a strong correlation between gross pathological alterations and histopathological findings on the infiltration of inflammatory cells and epidermal erosion. Furthermore, the findings of this study show that the severity of both gross and microscopic alterations correlates with a net increase in bacterial growth at the skin. Previous studies have shown that at an early stage of infection with virulent GAS (48 h post-inoculation) the stratum corneum is lost over large sections of skin, and single cocci or short chains are observed in close association with the newly exposed apical surface of the viable keratinocytes; furthermore, the number of tissue-associated bacteria is low and few PMNs are present (Scaramuzzino et al., 2000). We propose that at this early stage of infection, the bacteria are in a nutrient-poor environment (Fig. 4). A nutrient-poor environment is consistent with a stationary phase of growth and maximal expression of cysteine protease activity due to SpeB (Lyon et al., 1998; Podbielski et al., 1999; Svensson et al., 2000). 


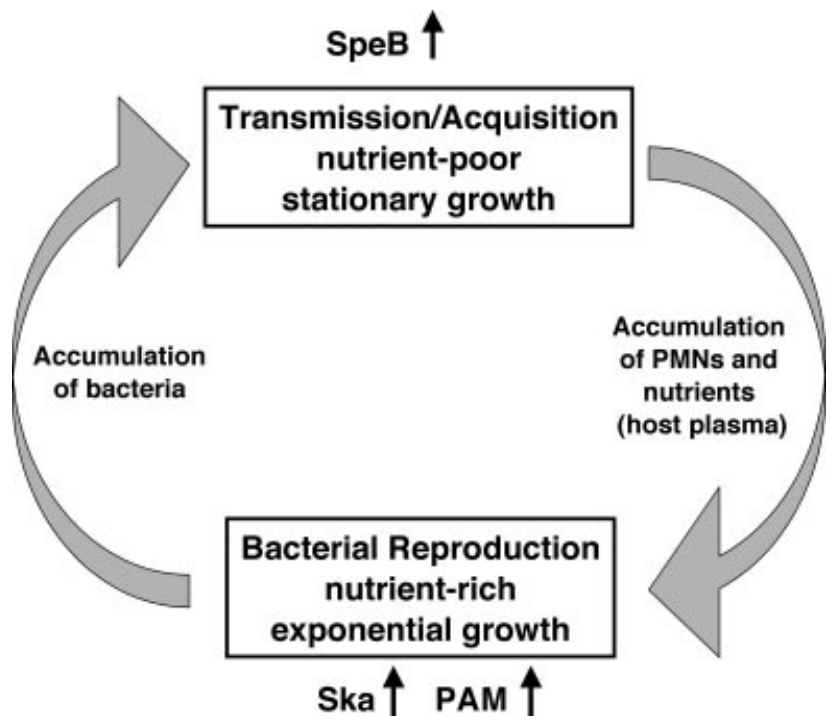

Fig. 4. Integrated model for the pathogenesis of GAS impetigo. It is proposed that SpeB acts at an early stage of infection and is involved in the early amplification of the inflammatory response, whereby PMNs and plasma begin to accumulate in the vicinity of the bacteria adherent to the apical surface of keratinocytes. Loss of SpeB prevents the inflammatory response and bacteria fail to replicate. Host plasma that enters the infection site constitutes an important source of nutrients, leading to bacterial reproduction. Through the combined action of streptokinase and PAM, bacterial-generated plasmin activity leads to fibrinolysis, which, in turn, prevents the skin lesions from drying out and healing. Through fibrinolytic activity, the window of opportunity for a continuing inflammatory infiltrate and ensuing bacterial reproduction is greatly expanded.

Inactivation of $s p e B$ leads to complete attenuation of virulence in the experimental model for impetigo (Svensson et al., 2000), suggesting that SpeB plays a critical role during early stages of infection, prior to extensive bacterial growth.

By 4 days post-inoculation with virulent GAS, the external surface of the epidermis has been transformed into a nutrient-rich environment (Scaramuzzino et al., 2000). Extravasating PMNs drag along small amounts of host plasma in their migration from dermal vessels to the outer epidermal surface, wherein both PMNs and plasma gradually accumulate to form a copious purulent exudate (pus) that is visible by gross pathology. Some bacterial growth has occurred by 4 days, as evidenced by both a net increase in c.f.u. counts and readily visible, large aggregates of cocci at the outer rim of the pus layer (Scaramuzzino et al., 2000). The massive aggregates of cocci appear to be well-positioned for transmission to new hosts and, because of their high density, resistance to phagocytosis may result from steric hindrance.

Extracellular streptokinase is produced during exponential growth (Fig. 2); therefore, streptokinase appears to be essential at some time during the nutrient-rich phase of infection (Fig. 4). Host Plg is (presumably) present in the local micro-environment (i.e. purulent exudate) and its activation by streptokinase appears to be critical for ensuing bacterial reproduction, although incomplete attenuation of the ALAB49 $\Delta s k a$ mutant suggests that limited bacterial growth (or survival) can occur in the absence of streptokinase. If streptokinase is critical during the earliest stage of the nutrient-rich phase of infection, it may act, directly or indirectly, to further amplify the inflammatory response, leading to the recruitment of additional PMNs and host plasma. Bacterial-generated plasmin activity can lead to fibrinolysis, which, in turn, may prevent the skin lesion from drying out and healing. If streptokinase plays a role during the late stages of the nutrient-rich phase of infection, it may act to promote invasion of GAS into the host tissue. Unlike PAM, streptokinase does not appear to directly contribute to resistance to phagocytosis, based on findings with the in vitro bactericidal assay. By 7 days post-inoculation, bacterial reproduction has increased even further and dermal pockets of Grampositive cocci are readily observed (Scaramuzzino et al., 2000).

It is proposed that PAM exerts its virulence properties during the nutrient-rich phase of infection (Fig. 4), since $\mathrm{emm}$ gene transcription is up-regulated during the exponential phase (McIver \& Scott, 1997). It is not known whether the slight attenuation observed for ALAB49 4 pam in vivo is related to the partial loss in its antiphagocytic property or to the decreased bacterialbound plasmin activity in vitro. The ALAB49 sska mutant exhibits a greater loss in virulence than the ALAB49 $\Delta$ pam mutant, suggesting that PAM function can be partly compensated by low-affinity Plg-binding proteins present on the bacterial surface (Lottenberg et al., 1992; Pancholi \& Fischetti, 1992, 1998) and/or by fluid-phase plasmin activity. Since the affinity of PAM for mouse Plg is 10- to 20 -fold lower than its human counterpart, the contribution of PAM to virulence may be delayed until additional plasma is dragged to the epidermal surface by extravasating PMNs. A possible consequence for the hu-skin-SCID mouse model is that the minimal infective dose is somewhat higher than for natural infection in humans.

As ALAB49 re-enters another round of stationary growth, the increased expression of cysteine protease activity due to SpeB results in the degradation of extracellular streptokinase. It is not yet known whether streptokinase is deleterious to GAS transmission and/or the early stages of a newly acquired infection. Conceivably, bacterial-generated plasmin might act to degrade streptococcal adhesins or host keratinocyte receptors that are critical for the initial host-pathogen interaction. Alternatively, SpeB degradation of streptokinase might simply be incidental.

Although the ALAB49 sska and $\Delta p a m$ mutants are only partially attenuated in the experimental model for impetigo, it is quite possible that in the natural world comparable mutants would become completely lost. In a human host population, GAS infectious doses are 
probably at the lower end of our experimental range. In order for a micro-organism to survive, its basic reproductive rate $\left(R_{0}\right)$ must be greater or equal to one (Anderson \& May, 1991; Anderson, 1998), which essentially means that for each infected host that recovers, the organism must be successfully transmitted to at least one new host. If bacterial doses are naturally low and the mutated variant can induce infection in only a fraction of newly exposed hosts, the organism will quickly disappear.

From the standpoint of evolutionary success, changes in c.f.u. counts are highly relevant measures. Fitness is defined in the context of the environmental niche, and the organism with the highest fitness is that which yields the greatest number of progeny in that niche (i.e. it is the most highly adapted). An increase in the number of (transmissible) progeny increases the frequency of adaptive alleles in the gene pool of that subpopulation. The finding that the PAM phenotype and high levels of SpeB activity display statistically significant associations with emm pattern D strains and impetigo isolates (Svensson et al., 1999, 2000) is consistent with the idea that they play a direct role in promoting skin infection. In addition to $\mathrm{emm}$ pattern D strains, emm pattern E strains of GAS are often associated with impetigo, although they are prevalent at the URT as well (Bessen et al., 1996, 2000; Dicuonzo et al., 2001). To date, pattern E strains have not been carefully investigated in the hu-skin-SCID mouse model. Since emm pattern E strains lack PAM (Svensson et al., 1999), it is possible that they cause impetigo by using an entirely different molecular strategy than pattern $\mathrm{D}$ strains.

Following mutagenesis of any gene, unintended polar effects can potentially arise, not only through the introduction of strong transcriptional terminators within the $\Omega \mathrm{Km}-2$ element (as is the case for the ALAB49 $\Delta s k a$ and $\Delta$ pam mutants) but also through indels generated by allelic replacement and ensuing shifts in codon usage and translational activity. In many respects, an ideal control for the ALAB49 $\Delta s k a$ and $\Delta$ pam mutants is the reintroduction of the wild-type gene by transcomplementation, although there is a downside lying in variable gene dosage effects. Transcomplementation was achieved for the ALAB49 $\Delta$ pam mutant, and the binding activity of human Plg to whole bacteria, when tested in vitro, was fully restored (data not shown). However, when this construct was tested in the hu-skin-SCID mouse model for impetigo, a confusing picture emerged whereby gross and microscopic pathological alterations were evident in the absence of net bacterial growth at the skin. These findings are probably an artefact of plasmid instability resulting from shifts in selective pressures that occur during the course of infection, i.e. uneven diffusion of the selectable antibiotic to the superficial tissue site and changing needs for PAM. Once the plasmid is lost due to a transient lapse in positive selection, the bacteria can be eliminated following subsequent exposure to antibiotics or PMNs. Despite the lack of this particular control, the numerous phenotypic tests performed in vitro provide strong support that the ALAB49 $\Delta s k a$ and $\Delta$ pam mutants did not undergo unintended alterations in several phenotypes that are known to affect virulence. Although the $\Omega \mathrm{Km}-2$ element contains strong transcriptional terminators, both ska and emm genes are expressed as monocistronic transcripts, and the ORF transcribed on the strand opposite to $s k a$ shares a bidirectional transcriptional terminator positioned downstream of $s k a$ (Bessen \& Fischetti, 1992; Malke, 2000; Malke et al., 2000; Podbielski et al., 1995; Yung \& Hollingshead, 1996); therefore, expression of adjacent genes should be unaffected. In summary, there is no evidence that the $\mathrm{ALAB} 49 \Delta s k a$ and $\Delta$ pam mutants have alterations in other genes.

It has been proposed that bacterial-generated plasmin acts to modulate the micro-environment of the bacterium and its movement through host tissue (Boyle \& Lottenberg, 1997; Coleman et al., 1997; Rasmussen \& Bjorck, 2002; Sodeinde et al., 1992). However, the ability to spread into deep tissue and cause invasive disease does not appear to be a long-term evolutionary strategy for GAS. The URT and epidermis of the skin are the primary tissue reservoirs for GAS. Because infection at the throat and skin is typically mild and superficial, the infected host is fairly mobile and has a high potential for exposing numerous individuals, via respiratory droplets or direct contact. Since GAS undergo expansive reproductive growth at the superficial epithelium and transmission to new hosts is launched from the superficial epithelium as well, it is at the URT and epidermis where the evolution of GAS is most profoundly shaped by the forces of natural selection. Thus, it seems most likely that the primary function of bacterial-generated plasmin activity is to enhance survival at a superficial epithelial site, as opposed to deep tissue.

The epidemiological findings, in combination with the experimental data, make a strong case for a role for both extracellular plasmin (this study) and cysteine proteinase activities (Svensson et al., 2000) as key determinants of tissue tropism at the skin. An interesting aspect of this finding is that one usually envisions determinants of host or tissue range as attachment factors that recognize a specific host-cell receptor, yet, here, extracellular enzymes appear to play that role. By modulating their local micro-environment through proteolytic pathways, GAS have been highly successful in exploiting this ecological niche.

\section{ACKNOWLEDGEMENTS}

The authors thank Christy Schilling, Malgorzata Berlikowski and David Chu for their technical assistance, Awdhesh Kalia for PCR-based mapping of the ska chromosomal region in wild-type strain ALAB49 and Horst Malke for providing antiserum to streptokinase. This work was supported by the National Institutes of Health (R01-AI28944 and R01GM60793, to D.E.B.), the American Heart Association, a Pilot/Feasibility award from the Yale Skin Diseases Research Center (supported by P30-AR-041942), the Swedish Research 
Council (9926), and the Crafoord, Kock and Österlund Foundations. M.D.S. is the recipient of a graduate scholarship from the Foundation for Strategic Research (Infection and Vaccinology programme).

\section{REFERENCES}

Anderson, R. \& May, R. (1991). Infectious Diseases of Humans: Dynamics and Control. Oxford: Oxford University Press.

Anderson, R. M. (1998). Analytic theory of epidemics. In Emerging Infections, pp. 23-50. Edited by R. M. Krause. New York: Academic Press.

Anthony, B. F., Kaplan, E. L., Wannamaker, L. W. \& Chapman, S. S. (1976). The dynamics of streptococcal infections in a defined population of children: serotypes associated with skin and respiratory infections. Am J Epidemiol 104, 652-666.

Ashbaugh, C. D. \& Wessels, M. R. (2001). Absence of a cysteine protease effect on bacterial virulence in two murine models of human invasive group A streptococcal infection. Infect Immun 69, 6683-6688.

Berge, A. \& Björck, L. (1995). Streptococcal cysteine proteinase releases biologically active fragments of streptococcal surface proteins. J Biol Chem 270, 9862-9867.

Berge, A. \& Sjöbring, U. (1993). PAM, a novel plasminogenbinding protein from Streptococcus pyogenes. J Biol Chem 268, 25417-25424

Bessen, D. E. \& Fischetti, V. A. (1992). Nucleotide sequences of two adjacent $M$ and $M$-like protein genes of group A streptococci: different RNA transcript levels and identification of a unique IgAbinding protein. Infect Immun 60, 124-135.

Bessen, D. E., Sotir, C. M., Readdy, T. L. \& Hollingshead, S. K. (1996). Genetic correlates of throat and skin isolates of group A streptococci. J Infect Dis 173, 896-900.

Bessen, D. E., Carapetis, J. R., Beall, B. \& 7 other authors (2000). Contrasting molecular epidemiology of group A streptococci causing tropical and non-tropical infections of the skin and throat. J Infect Dis 182, 1109-1116.

Bisno, A. L. \& Stevens, D. (2000). Streptococcus pyogenes (including streptococcal toxic shock syndrome and necrotizing fasciitis). In Principles and Practice of Infectious Diseases, pp. 2101-2117. Edited by G. L. Mandell, R. G. Douglas \& R. Dolin. Philadelphia: Churchill Livingstone.

Boyle, M. \& Lottenberg, R. (1997). Plasminogen activation by invasive human pathogens. Thromb Haemostasis 77, 1-10.

Caparon, M. (2000). Genetics of group A streptococci. In GramPositive Pathogens, pp. 53-65. Edited by V. A. Fischetti, R. P. Novick, J. J. Ferretti, D. A. Portnoy \& J. I. Rood. Washington, DC: American Society for Microbiology.

Coleman, J., Gebbia, J., Piesman, J., Degen, J., Bugge, T. \& Benach, J. (1997). Plasminogen is required for efficient dissemination of $B$. burgdorferi in ticks and for enhancement of spirochetemia in mice. Cell 89, 1111-1119.

Collin, M. \& Olsen, A. (2000). Generation of a mature streptococcal cysteine proteinase is dependent on cell wall-anchored M1 protein. Mol Microbiol 36, 1306-1318.

Cunningham, M. W. (2000). Pathogenesis of group A streptococcal infections. Clin Microbiol Rev 13, 470-511.

Darmstadt, G., Mentele, L., Podbielski, A. \& Rubens, C. (2000). Role of group A streptococcal virulence factors in adherence to keratinocytes. Infect Immun 68, 1215-1221.

Dicuonzo, G., Gherardi, G., Lorino, G., Angeletti, S., DeCesaris, M., Fiscarelli, E., Bessen, D. E. \& Beall, B. (2001). Group A streptococcal genotypes from pediatric throat isolates in Rome, Italy. J Clin Microbiol 39, 1687-1690.

Enright, M. C., Spratt, B. G., Kalia, A., Cross, J. H. \& Bessen, D. E. (2001). Multilocus sequence typing of Streptococcus pyogenes and the relationship between $\mathrm{emm}$-type and clone. Infect Immun 69, 2416-2427.

Feil, E. J., Holmes, E. C., Bessen, D. E. \& 9 other authors (2001). Recombination within natural populations of pathogenic bacteria: short-term empirical estimates and long-term phylogenetic consequences. Proc Natl Acad Sci U S A 98, 182-187.

Ferretti, J. J., McShan, W. M., Ajdic, D. \& 20 other authors (2001). Complete genome sequence of an M1 strain of Streptococcus pyogenes. Proc Natl Acad Sci U S A 98, 4658-4663.

Fischetti, V. (2000). Surface proteins on gram positive bacteria. In Gram Positive Pathogens, pp. 11-24. Edited by V. A. Fischetti, R. P. Novick, J. J. Ferretti, D. A. Portnoy \& J. I. Rood. Washington, DC: American Society for Microbiology.

Geyer, A. \& Schmidt, K. H. (2000). Genetic organisation of the M protein region in human isolates of group $\mathrm{C}$ and $\mathrm{G}$ streptococci: two types of multigene regulator-like $(m g r C)$ regions. Mol Gen Genet 262, 965-976.

Husmann, L. K., Scott, J. R., Lindahl, G. \& Stenberg, L. (1995). Expression of the Arp protein, a member of the M protein family, is not sufficient to inhibit phagocytosis of Streptococcus pyogenes. Infect Immun 63, 345-348.

Johnson, D., Kaplan, E., Sramek, J., Bicova, R., Havlicek, J., Havlickova, H., Motlova, J. \& Kriz, P. (1996). Laboratory Diagnosis of Group A Streptococcal Infections. Geneva: World Health Organization.

Johnston, K. H. \& Zabriskie, J. B. (1986). Purification and partial characterization of the nephritis strain-associated protein from Streptococcus pyogenes, group A. J Exp Med 163, 697-712.

Kalia, A., Spratt, B. G., Enright, M. C. \& Bessen, D. E. (2002). Influence of recombination and niche separation on the population genetic structure of the pathogen Streptococcus pyogenes. Infect Immun 70, 1971-1983.

Kotarsky, H., Thern, A., Lindahl, G. \& Sjobring, U. (2000). Strainspecific restriction of the antiphagocytic property of group A streptococcal M proteins. Infect Immun 68, 107-112.

Lottenberg, R., Broder, C. C., Boyle, M. D. P., Kain, S. J., Schroeder, B. L. \& Curtiss, R. I. (1992). Cloning, sequence analysis, and expression in Escherichia coli of a streptococcal plasmin receptor. J Bacteriol 174, 5204-5210.

Lyon, W., Gibson, C. \& Caparon, M. (1998). A role for trigger factor and an Rgg-like regulator in the transcription, secretion and processing of the cysteine protease of Streptococcus pyogenes. EMBO J 17, 6263-6275.

Malke, H. (2000). Genetics and pathogenicity factors of group C and G streptococci. In Gram-Positive Pathogens, pp. 163-176. Edited by V. A. Fischetti, R. P. Novick, J. J. Ferretti, D. A. Portnoy \& J. I. Rood. Washington, DC: American Society for Microbiology.

Malke, H., Steiner, K., Gase, K. \& Frank, C. (2000). Expression and regulation of the streptokinase gene. Methods 21, 111-124.

Maxted, W. R. (1980). Disease association and geographical distribution of the $M$ types of group $A$ streptococci. In Streptococcal Diseases and the Immune Response, pp. 763-777. Edited by S. E. Read \& J. B. Zabriskie. New York: Academic Press.

Mclver, K. S. \& Scott, J. R. (1997). Role of $m g a$ in growth phase regulation of virulence genes of the group A streptococcus. $J$ Bacteriol 179, 5178-5187. 
Pancholi, V. \& Fischetti, V. A. (1992). A major surface protein of group A streptococci is a glyceraldehyde-3-phosphate-dehydrogenase with multiple binding activity. J Exp Med 176, 415-426.

Pancholi, V. \& Fischetti, V. A. (1998). Alpha-enolase, a novel strong plasmin(ogen) binding protein on the surface of pathogenic streptococci. J Biol Chem 273, 14503-14515.

Perez-Casal, J., Price, J. A., Maguin, E. \& Scott, J. R. (1993). An M protein with a single $C$ repeat prevents phagocytosis of Streptococcus pyogenes: use of a temperature-sensitive shuttle vector to deliver homologous sequences to the chromosome of $S$. pyogenes. Mol Microbiol 8, 809-819.

Podbielski, A., Flosdorff, A. \& Weber-Heynemann, J. (1995). The group A streptococcal virR49 gene controls expression of four structural vir regulon genes. Infect Immun 63, 9-20.

Podbielski, A., Schnitzler, N., Beyhs, P. \& Boyle, M. (1996). Mrelated protein (Mrp) contributes to group A streptococcal resistance to phagocytosis by human granulocytes. Mol Microbiol 19, 429-441.

Podbielski, A., Woischnik, M., Kreikemeyer, B., Bettenbrock, K. \& Buttaro, B. A. (1999). Cysteine protease SpeB expression in group A streptococci is influenced by the nutritional environment but SpeB does not contribute to obtaining essential nutrients. Med Microbiol Immunol 188, 99-109.

Raeder, R., Woischnik, M., Podbielski, A. \& Boyle, M. D. (1998). A secreted streptococcal cysteine protease can cleave a surfaceexpressed M1 protein and alter the immunoglobulin binding properties. Res Microbiol 149, 539-548.

Rasmussen, M. \& Bjorck, L. (2002). Proteolysis and its regulation at the surface of Streptococcus pyogenes. Mol Microbiol 43, 537-544.

Ringdahl, U., Svensson, M., Wistedt, A., Renné, T., Kellner, R., Müller-Esterl, W. \& Sjöbring, U. (1998). Molecular co-operation between protein PAM and streptokinase for plasmin acquisition by Streptococcus pyogenes. J Biol Chem 273, 6424-6430.
Scaramuzzino, D. A., McNiff, J. M. \& Bessen, D. E. (2000). Humanized in vivo model for streptococcal impetigo. Infect Immun 68, 2880-2887.

Schrager, H., Alberti, S., Cywes, C., Dougherty, G. \& Wessels, M. (1998). Hyaluronic acid capsule modulates $M$ protein-mediated adherence and acts as a ligand for attachment of group A streptococcus to CD44 on human keratinocytes. J Clin Investig 101, 1708-1716.

Scott, J. R., Guenther, P. C., Malone, L. M. \& Fischetti, V. A. (1986). Conversion of an $\mathrm{M}^{-}$group A streptococcus to $\mathrm{M}^{+}$by transfer of a plasmid containing an M6 gene. J Exp Med 164, 1641-1651.

Sodeinde, O., Subrahmanyam, Y., Stark, K., Quan, T., Bao, Y. \& Goguen, J. (1992). A surface protease and the invasive character of plague. Science 258, 1004-1007.

Svensson, M. D., Sjöbring, U. \& Bessen, D. E. (1999). Selective distribution of a high-affinity plasminogen binding site among group A streptococci associated with impetigo. Infect Immun 67, 3915-3920.

Svensson, M. D., Scaramuzzino, D. A., Sjobring, U., Olsen, A., Frank, C. \& Bessen, D. E. (2000). Role for a secreted cysteine proteinase in the establishment of host tissue tropism by group A streptococci. Mol Microbiol 38, 242-253.

Wannamaker, L. W. (1970). Differences between streptococcal infections of the throat and of the skin. N Engl J Med 282, 23-31.

Yung, D.-L. \& Hollingshead, S. K. (1996). DNA sequencing and gene expression of the emm gene cluster in an M50 group A streptococcus strain virulent for mice. Infect Immun 64, 2193-2200.

Received 25 July 2002; revised 12 August 2002; accepted 11 September 2002. 